\title{
Study on a Novel Capacitive Pressure Sensor with Large Dynamic Range Zhang Tongtong $^{1,2, a}$, Zhang Zhengyuan ${ }^{2, b}$ \\ ${ }^{1}$ Chongqing University of Posts and Telecommunications, Chongqing 400060, P.R. China \\ ${ }^{2}$ National Laboratory of Analog Integrated Circuits, Chongqing 400060, P.R. China \\ aemail: today_ztt@163.com, bemail: zhangzy456789@163.com
}

Keywords: capacitive pressure sensor; circular flat diaphragm; large dynamic range

\begin{abstract}
In this paper, a novel capacitive pressure sensor with large dynamic range is presented. The working principle and the design structure of the sensor are demonstrated in the paper, then the theoretical calculation of mechanical properties, capacitance and the simulate results are performed. The study shows that the sensitivity of this capacitive pressure transducers is about $0.094 \mathrm{f} \mathrm{F} / \mathrm{h} \mathrm{Pa}$ over the range of $20 \sim 100 \mathrm{k} \mathrm{Pa}$. The advantage of the structure is that it has a large dynamic range, higher tolerance against the environment than others and the measure flexible according to the demand.
\end{abstract}

\section{Introduction}

Pressure sensors are more and more popular in many fields, such as medical applications and industrial control, and among the all kinds of pressure sensors, capacitive pressure sensors are most outstanding. Comparing with the piezoresistive sensor, this kind of sensor has many advantages, low noise in very low power applications, low temperature hysteresis and high tolerance against overpressure situations and high sensitivity ${ }^{[1]}$, which makes it has a good prospect in the industry. According to the capacitive equation: $\mathrm{C}=\varepsilon \frac{A}{d}$, the capacitance will change as a result of the variation of the displacement between the two electrodes, the permittivity and the area.

This paper demonstrates a new structure of capacitive pressure sensor, whose capacitance vary according to the variation of separation distance result from the pressure applied on the electrode. The most outstanding of this kind of capacitive pressure sensor is the large dynamic range. Besides, the electrode poles are designed on the same side, which makes the sensor less sensitive to the environment after being packaged than other kind of capacitive pressure sensor. What's more, the measure could be easily changed according to the special requirement. In the study, the design, fabrication process, and the calculation result are presented.

\section{Structure design}

The cross section of the capacitive pressure sensor is shown in Figure 1. This sensor consists of thin wafer and the thick wafer, which are top and bottom electrodes respectively. The top membrane is deformed under pressure, leading to a deflection bending, and hence causing the changes of the displacement between the electrodes.

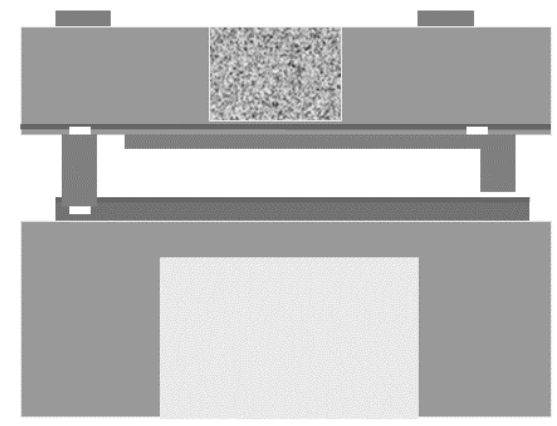

Figure 1 Cross section of the capacitive sensor

The capacitance of a parallel plate capacitor is given by the well-known equation is: 


$$
C_{0}=\varepsilon \frac{A}{d_{0}}
$$

Where $\mathrm{C}_{0}$ is the prime capacitance, $\varepsilon$ is the permittivity of the dielectric medium, $\mathrm{A}$ is the surface area of the electrodes, and $\mathrm{d}_{0}$ is the thickness of the dielectric ${ }^{[1]}$.

According to the theory of plates and shells, when the up electrode suffers the pressure $\mathrm{p}, \mathrm{h}$ represents the thickness of the elastic membrane and the radius is a, the circular flat diaphragm equation is ${ }^{[2]}$ :

$$
\begin{aligned}
& W_{r}=\frac{3 p\left(1-u^{2}\right)}{16 E h^{a}}\left(a^{2}-r^{2}\right)^{2} \\
& W_{\max }=W_{0}=\frac{p a^{4}}{64 D}
\end{aligned}
$$

Where $\mathrm{W}_{\mathrm{r}}$ is the deflection of the membrane, $\mathrm{u}$ is the Poisson ratio of the material, which is about 0.28 for Si, and Young's modulus $\mathrm{E}$ is about $1.76 \times 10^{11} \mathrm{~N} / \mathrm{m}^{2}$, and the flexural rigidity of the plate $\mathrm{D}=\frac{E h^{\mathrm{s}}}{12\left(1-v^{2}\right)}$.

Combined (1) and (2), it is easy to figure out that the capacitance due to the additivity of electric capacity is:

$$
\mathrm{C}=\int_{0}^{R} \frac{2 \pi \varepsilon}{d-W_{r}} \times r d r
$$

A great advantage of this pressure sensor is that the measure of the sensor could be adjusted according to the different demand. The requirements of large range or sensitivity could be met by changing the thickness of the membrane. Thus, the size below is just for analysis the performance of the structure. In consideration of the size and the application of the sensor, we determined that the space between the electrodes is $20 \mathrm{um}$, and the diameter of the circular flat is $1100 \mathrm{um}$, and the pressure applied on the membrane is range from $20 \sim 100 \mathrm{k} \mathrm{Pa}$. Figure 2 shows the relevance between thickness of the membrane and the maximum deformation at the pressure of $100 \mathrm{k} \mathrm{Pa}$.

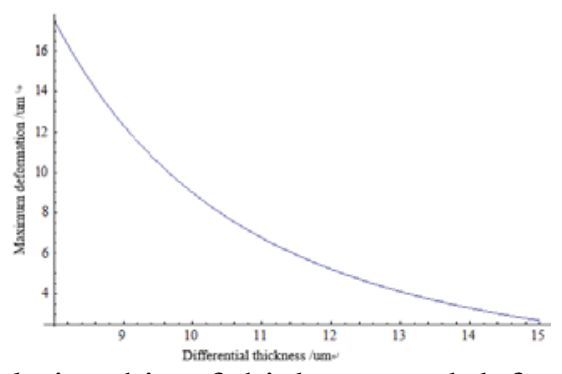

Figure 2 Relationship of thickness and deformation

On the basis of theory of thin plates, decreasing the thickness could improve the sensitivity of the sensor, at the same time, it will also weaken the strength of the membrane ${ }^{[3]}$. Taking all of these factors into consideration, we determine the measure of the thickness of elastic membrane is 10 um. Using ANSYS to build the entity model of sensor and mesh the model. Then, carry the boundary condition and pressure load ${ }^{[1]}$. The Figure 3 gives the deformation of the membrane under the pressure $100 \mathrm{k} \mathrm{Pa}$, and the Figure 4 shows the stress distribution with the same pressure. It is easily to find out that the largest deformation is small than the distance between the plates, and the maximum stress which is distributed at the edge of the circle is $0.1732 \mathrm{G} \mathrm{Pa}$, and it is far smaller than the yield strength of $\mathrm{Si}^{[3]}$.

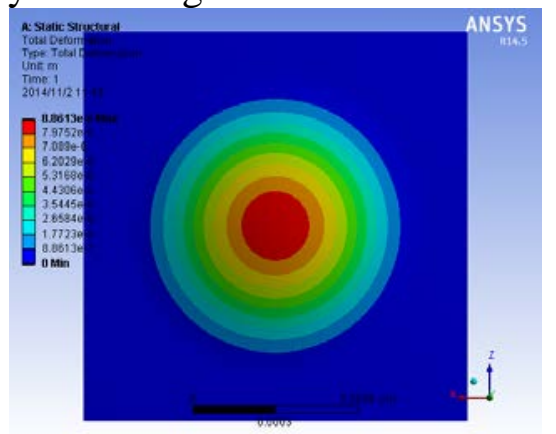

Figure 3 Deformation under 100k Pa

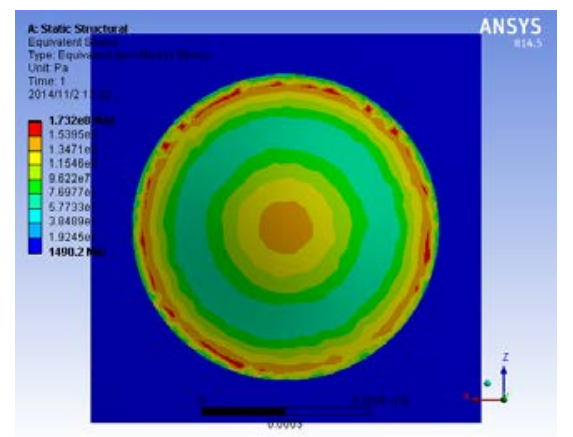

Figure 4 Stress distribution under 100k Pa 
A typical pressure response of the sensor with differential pressure is shown in the Figure 5. The pressure of Figure 5 (a) is ranging from $0 \mathrm{~Pa}$ to $100 \mathrm{k} \mathrm{Pa}$, and the pressure of Figure 5 (b) grows to 222612Pa, when the membrane is bended almost contact the bottom electrode, which is the maximum deformation in theory. It is obvious that the capacitive pressure transducer has a better linearity under the pressure of $150 \mathrm{k} \mathrm{Pa}$, and the good linearity is one of the guarantees of a better performance in some way.

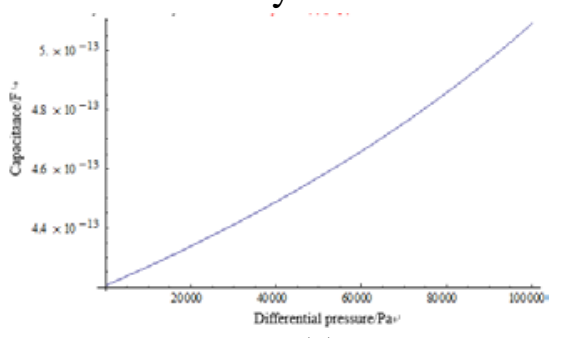

(a)

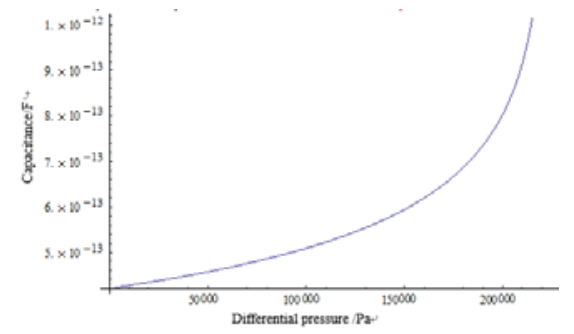

(b)

Figure 5 Curves of the capacitance with pressure

\section{Fabrication process}

The sensor is consisted of two parts: the thin wafer and the thick wafer. Thin wafer is diaphragm side, and thick wafer includes static electrode and insulating glass. The two parts are manufactured in separate batches, and the combination of them is made by anodic bonding. The entire fabrication is combined dry etching with wet etching ${ }^{[4]}$. The detail process flow shown in Figure 6 is listed at follows:

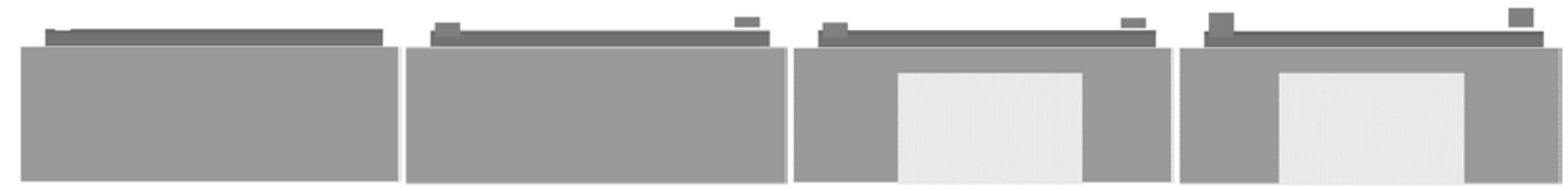

(a)

(b)

(c)

(d)

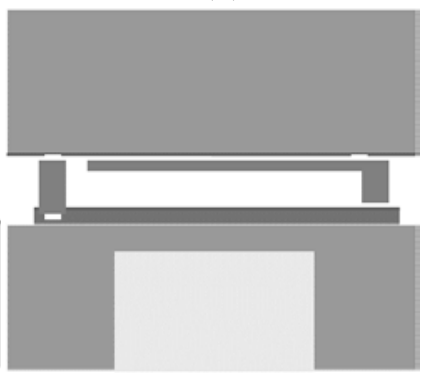

(e)

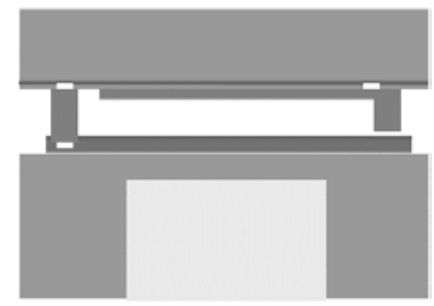

(i) (g)

(f)
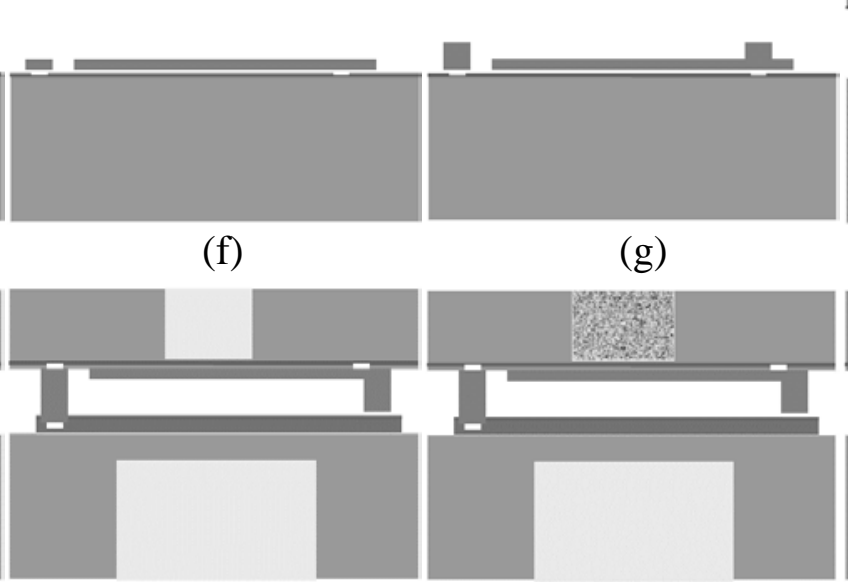

(j)

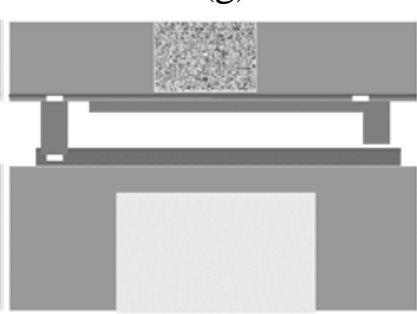

$(\mathrm{k})$ (h)

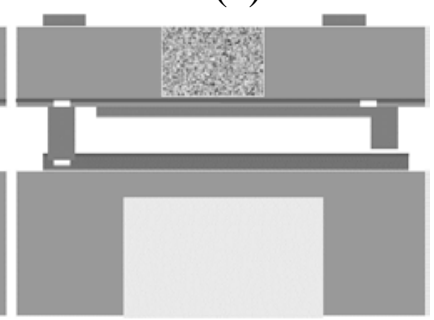

(l)

Figure 6 Fabrication process

(a) Grow thermal oxide on the thin wafer by dry method, which is used as a dielectric layer, and the thickness is about $0.15 \mathrm{um}$. And then lithography the layer.

(b) Sputtering mental and lithography the top electrode.

(c) Etch at the bottom of the silicon.

(d) Electroplate at the front side of the wafer.

(e) Grow oxide on the thick wafer, and lithography the layer.

(f) Sputtering mental and lithography the bottom electrode.

(g) Electroplate on the frontage of the silicon. 
(h) Combined the thin wafer with the thick wafer by anodic bonding.

(i) Reduce the thickness of the wafer and polish the surface of the silicon.

(j) Lithography the thick wafer.

(k) Fill the groove with glass and polish the surface.

(l) Sputtering mental and lithography to create electrodes and leads.

\section{Conclusion}

In this study, a novel capacitive pressure sensor is presented. The sensing capacitor pressure consisted of complex membranes ${ }^{[5]}$. The sensor introduced in Refs. [6] has a small operating range, and the transducer in [7] has the problem of the extraction of electrode pole. The improved structure presented in this study overcome these disadvantages ${ }^{[5]}$. This paper gives a theoretical analysis on silicon membrane mechanics characteristics and the deformation equation of circle membrane with pressure, and defines a sample measure of the sensor. A finite element model was also built by ANSYS for the design of the sensor.

The next step would be further development of the electric circuit to detect the tiny change of capacitance and have a test about the sensor.

\section{Acknowledgement}

In this paper, the research was sponsored by the National High-tech R\&D Program (863 Program) (Project No. 2012AA041201).

\section{References}

[1] Ezzat G. Bakhoum, Marvin H. M Cheng. Capacitive Pressure Sensor with Very Large Dynamic Range [J], 2010.

[2] Timoshenko S P, Krieger S W. Theory of plates and shells [M]. $2^{\text {nd }}$ edition. New York; McGraw-Hillm, 1970.

[3] HUANG Xiao-dong, WANG Bin, QIN Ming, et al. Design, fabrication and test of a monolithic capacitive pressure sensor [J]. Chinese Journal of Sensors and Actuators, 2008, 21 (4): 578-580.

[4] Tai-ran Hsu, MEMS and Microsystems: Design and Manufacture [M]. Boston; McGraw-Hillm, 2002.

[5] HUANG Xiao-dong, Huang Jian-qiu, QIN Ming, et al. A Novel Capacitive Pressure Sensor [J]. Journal of Semiconductors, 2008, 29 (3): 428-432.

[6] TAO Ye, ZENG Yi-bo, et al. Design and Fabrication of a Silicon Membrane for Micro Capacitive Pressure Sensors [J]. Journal of Xiamen University (Natural Science), 201352 (5) 627-632.

[7] Hussam Eldin A. Elgamel, A simple and efficient technique for the simulation of capacitive pressure transducers [J], Sensor and Actuators.1999 77, 183-186 